\title{
Actividades motivacionales para aprendices en línea
}

Ana Ilva Capera Urrego ${ }^{1}$

\section{Resumen}

Esta reflexión busca en primera instancia examinar el significado de la motivación, por lo que se presentan diferentes definiciones del término y puntos de vista de algunos autores. A continuación se analiza el papel que juega la motivación en el campo educativo y su importancia en el proceso de aprendizaje en línea. Una vez establecida esta condición, se indaga acerca de estrategias pedagógicas que pueden ser utilizadas en actividades de aprendizaje para apoyar el desempeño de los estudiantes. Finalmente se presentan algunas sugerencias sobre el tipo de actividades motivacionales que pueden aplicarse a los aprendices en línea.

Partiendo de la importancia de la motivación como el combustible que mueve a las personas a realizar actividades para alcanzar las metas propuestas, en el proceso educativo es innegable su papel para el logro de un aprendizaje significativo. En la educación a distancia este interés se orienta además hacia la concreción de un aprendizaje autónomo, autodirigido y autorregulado, que dé respuesta a los problemas de deserción y baja participación de los aprendices, que precisamente les impiden alcanzar sus metas por falta de esfuerzo y perseverancia. Es aquí donde el papel del tutor en la metodología a distancia es el de un facilitador del proceso de aprendizaje y el de un motivador para promover el compromiso, la participación y el rendimiento de los estudiantes. Para ello debe utilizar recursos y estrategias atractivas adaptadas a las necesidades de los aprendices, y en este caso, actividades en línea que orienten elementos motivadores, los cuales son esenciales en el éxito de los ambientes de aprendizaje a distancia.

1 Ingeniera Química, Especialista en Ingeniería de Procesos de Alimentos y Biomateriales, Magister en Lingüística aplicada a la enseñanza del Inglés, Candidata a Doctora en Tecnología Instruccional y Educación a Distancia. Actualmente docente auxiliar de la Escuela de Ciencias Básicas, Tecnología e Ingeniería de la Universidad Nacional Abierta y a Distancia (UNAD), Colombia. E-mail:ana.capera @unad.edu.co. 
Palabras clave: motivación, aprendizaje en línea, aprendizaje autónomo, educación a distancia, tutor, deserción.

\begin{abstract}
In the first place, this reflection seeks to examine the meaning of the motivation, so here you will find different definitions of the term and points of view of some authors. Then it examines the role of motivation in the field of education and its importance in the on line learning process. Once this condition is considered, it looks into teaching strategies that can be used in learning activities to support student performance. Finally, some suggestions on the type of motivational activities that may apply to online learners are presented.

Based on the importance of motivation as the fuel that moves people to carry out activities to achieve the proposed goals, its role in the achievement of a significant learning in the educational process is undeniable. In distance education, this interest is focused toward the realization of autonomous, self-directed and self-regulated learning, to answer problems of drop out and low participation of learners, which prevent them to achieve their goals because of lack of effort and perseverance. It is here where the role of the tutor in distance methodology, is that of a facilitator of the learning process and a motivator to promote commitment, involvement and student performance. To get it, he or she must use resources and attractive strategies adapted to the needs of the learners, and in this case, online activities to guide motivational elements, which are essential in the success of the distance learning environments.
\end{abstract}

Key words: motivation, online learning, autonomous learning, distance education, tutor, drop-out.

Recibido: 12 de agosto de 2010 Aceptado: 15 de octubre de 2010

\title{
Introducción
}

En cualquier campo de la vida el ser humano necesita un combustible, una fuerza o energía que lo mueva a realizar una actividad y a alcanzar un objetivo propuesto. El logro de objetivos y metas es directamente proporcional a las circunstancias favorables que conducen a ellos, al esfuerzo que se realiza y al afán por obtenerlos (Guzmán García, 2007). Pero lo que se quiere, la manera como se desea hacer y el tiempo a emplear, permiten buscar y definir el camino para llegar a ello. Según Guzmán García, todos los procesos y toma de 
decisiones que se hacen durante el transcurso de la vida dependen del grado de incentivación o motivación que posea la persona.

Si bien es cierto que dentro del ambiente educativo las actitudes, percepciones y expectativas que tiene el estudiante orientan su conducta, también es cierto que este contexto depende en gran medida de las acciones del profesor. De acuerdo con Díaz y Hernández (1998), el instructor es quien decide qué información presentar, cuándo y cómo hacerlo, qué objetivos proponer, qué actividades planear y cómo organizarlas, qué y cómo evaluar y cómo realimentar a los estudiantes. El docente ejerce una influencia decisiva en el proceso de enseñanza-aprendizaje, por lo que su motivación juega un papel importante, ya que lo conduce al desempeño de sus funciones con autonomía, perseverancia, iniciativa y compromiso hacia el mejoramiento de este proceso. Esta actitud orientada hacia el estudiante, va a generar en este un ambiente de motivación positiva hacia el estudio y su participación en dicho proceso. El grado y la calidad de motivación que un estudiante tenga, son, en consecuencia, básicos para su permanencia y retención durante su formación académica.

\section{Planteamiento del problema}

Si bien la educación a distancia es probablemente el área de mayor crecimiento de la educación a nivel internacional, sigue sufriendo de una debilidad fundamental y es la alta tasa de deserción de sus estudiantes, que es mayor si se compara con la educación convencional (Boyle, Kwon, Ross \& Simpson, 2010). De acuerdo con Yukselturk e Inan (2006), varios autores han reportado entre 1995 y 2001, a nivel internacional, tasas de deserción en la educación a distancia entre $30 \mathrm{y}$ $50 \%$. En Europa, por ejemplo, se encontraron índices de deserción en este tipo de programas, que varían entre 20 y $30 \%$, mientras que en Asia, las tasas reportadas fueron mayores de $50 \%$ y en Estados Unidos variaron entre 20 y $50 \%$

En el caso de Colombia, de acuerdo con Isaza (2009), el Ministerio de Educación Nacional (MEN) realizó varios estudios y encontró que de cada dos estudiantes que ingresan al sistema de educación superior tradicional, solamente uno se gradúa. Así entre los años 2002 y 2007 el MEN reportó tasas de deserción del $47 \%$, cercanas al promedio latinoamericano valorado para entonces en $50 \%$ (MEN, 2008). Este problema es mayor para la educación a distancia, donde según el Sistema de Prevención y Análisis de la Deserción en las Instituciones de Educación Superior, (SpadIES), dispositivo implementado por el MEN (2009), se encuentran tasas de deserción por cohorte que varían entre el 61 y el 52\% durante los años 2009 y 2010.

Según Isaza (2009), la deserción se ha convertido en uno de los mayores costos sociales e individuales en la educación superior en Colombia. Esto concuerda 
con Nichols (2010), quien afirma que uno de los mayores intereses tanto institucionales como del estudiante es su permanencia hasta finalizar el programa cursado, debido a los considerables costos que la deserción implica. Además de los costos financieros que afectan a ambos, existe un costo emocional real para el estudiante que abandona sus estudios y es que esa pérdida de confianza en sí mismo le impide continuar sus estudios (Simpson, 2004).

Con respecto a las causas de deserción y haciendo referencia específica a la educación a distancia, Yukselturk e Inan (2006) como resultado de una revisión de la literatura, las clasifican en cuatro categorías: barreras de los estudiantes como costos y motivación, retroalimentación y contacto con el profesor, apoyo y servicios para ellos, soledad y falta de experiencia; barreras de las facultades relacionadas con la falta de capacitación del personal en el desarrollo de cursos y uso de la tecnología, y falta de apoyo para el aprendizaje a distancia en general; barreras organizacionales, especialmente en infraestructura, disponibilidad de fondos y problemas tecnológicos; y consideraciones sobre el curso respecto a la evaluación del desempeño de los estudiantes y ritmo en el desarrollo de los cursos de programas a distancia.

En este sentido, Facundo (2009) realizó una investigación en la Universidad Nacional Abierta y a Distancia sobre la deserción, orientando el análisis de las causas hacia factores institucionales y personales. Los resultados obtenidos mediante la aplicación de una encuesta a un grupo de desertores, mostraron que con respecto a los primeros factores, el problema principal identificado se refería a la inter-relación académica: "la orientación y apoyo brindado por los consejeros y la atención de los tutores", quienes brindan "escasa atención al aprendizaje de los estudiantes" (p. 136). De este planteamiento y otros análisis realizados, Facundo concluye que se evidencia reiteradamente la deficiencia en la inducción y el proceso de motivación hacia la metodología a distancia y en la comunicación, atención y apoyo académico. Sobre los factores personales, la falta de disponibilidad de recursos financieros y de tiempo para estudiar, son las causas más significativas.

Partiendo de los resultados anteriores, esta reflexión se orienta hacia el tema de la falta de motivación, mencionada por Yukselturk e Inan (2006) y Facundo (2009), como uno de los principales factores de deserción.

\section{Desarrollo}

En la educación a distancia, de acuerdo con Pallof y Pratt ( Simonson, Smaldino, Albright \& Zvacek, 2009), el rol del profesor es el de un facilitador del proceso de aprendizaje y del logro de las metas educativas. Dentro de sus funciones está inmersa la de propiciar la motivación en los estudiantes, además de desarrollar las 
habilidades y estrategias que les permitan ser más competentes. Sumadas ellas a sus capacidades generales, su conocimientos previos y sus estilos de aprendizaje, se convierten en indicadores de logro para asegurar su éxito en la educación a distancia (Simonson et al.).

Sin embargo, como lo plantean Urdan y Schoenfelder (2006), en una investigación reciente se ha encontrado que la cognición de los individuos en relación con el trabajo académico, así como la fe en su capacidad y las expectativas sobre los resultados de su participación en las tareas, están influenciadas por factores socio-contextuales. Dentro de estos elementos pueden considerarse los mensajes del profesor acerca de la dificultad de la tarea, la capacidad percibida de los compañeros de clase, la información sobre la importancia de dominar las temáticas, y así sucesivamente. La motivación no reside por completo en el individuo o enteramente dentro del contexto. Más bien la motivación emerge de la interacción entre individuos dentro del entorno social del aula y de la escuela.

Dentro de este contexto se debe esperar que los estudiantes sientan que las actividades académicas son significativas y que valen la pena (Brophy, 2004). No puede esperarse que las vean divertidas, como juegos recreativos y pasatiempos. Incluso cuando ellos encuentran el contenido interesante y la actividad placentera, el aprendizaje requiere concentración y esfuerzo permanente. Los maestros pueden ayudar a los estudiantes a apreciar sus oportunidades de aprendizaje y a encontrar las actividades académicas significativas y de valor, mediante razones que incluyan motivación intrínseca y autorrealización.

Sin embargo, hasta aquí todas estas consideraciones están dirigidas hacia los ambientes tradicionales, pero ¿qué hay con respecto a la enseñanza a distancia? De acuerdo con Simonson et al.(2009), a pesar de que los estudiantes reportan su preferencia por la educación tradicional, están en forma creciente solicitando su admisión en cursos en línea o a distancia, debido a la conveniencia de la educación bajo esta modalidad, que a menudo sobrepasa otras consideraciones. No obstante, considerando por un momento el salón de clase tradicional, Dennen y Bonk (2007) se preguntan por qué los estudiantes asisten a clase. Quizá porque están interesados en el tópico o porque el profesor les está recordando su presencia. Y ¿por qué ellos participan en actividades de curso cara a cara? Para empezar, están sentados en el salón, por lo que pueden participar; adicionalmente, la actuación del instructor al presentar las actividades deseadas y la participación de los compañeros pueden motivar a los alumnos pasivos para que comiencen a ser un poco activos.

Las experiencias de aprendizaje son críticas para el éxito de la educación a distancia. La asistencia a la clase en línea es claramente diferente. A menos que de manera explícita se diga cómo se notará su asistencia, por ejemplo a través de mensajes enviados semanalmente, los estudiantes en línea no lo sabrán. La participación de aprendices en una clase en línea, algunas veces ha sido llamada un "acto de fe". (Salmon en Dennen \& Bonk, 2007). 
Con la proliferación de los sistemas de enseñanza a distancia, metodología que actualmente se ha convertido en la forma de educación de mayor crecimiento tanto a nivel nacional como internacional, (Gunawardena \& McIsaac, 2004), ha sido necesario buscar y conocer varios tipos de actividades de aprendizaje que puedan utilizarse para mejorar su calidad en línea. Tales actividades incluyen aprendizaje basado en problemas, aprendizaje basado en investigación, actividades de realimentación de compañeros, aprendizaje de casos en línea y debates en línea. Más allá de estas actividades existen estrategias pedagógicas que se pueden utilizar para apoyar el rendimiento de los estudiantes (Bonk \& Dennen, 2007). Para el aprendizaje en línea, se ha diseñado una serie de estrategias instruccionales para la Web ( Bonk \& Reynolds, en Bonk \& Dennen). Entre estas existen algunas que se relacionan con la motivación y revisten importancia dentro del proceso educativo.

\section{Análisis y discusión}

Dado que el término de motivación es tratado por varios autores desde diferentes perspectivas y diversos ámbitos, es necesario aclarar que para este trabajo se parte de un concepto general y luego se orienta hacia el campo de la psicología, sin profundizar más allá de los niveles intrínseco y extrínseco de su definición. En el nivel general se encuentran varias definiciones de esta palabra, todas relacionadas con su proveniencia del verbo latino movere, que significa "moverse", "poner en movimiento" o "estar listo para la acción” (Díaz \& Hernández, 1998, p. 35). A continuación se presentan algunos de los significados propuestos.

Según Woolfolk (1999), la motivación es un estado interno que incita, dirige y mantiene la conducta. Para Guzmán García (2007), la motivación es lo que garantiza que las personas estén enfocadas hacia lo que quieren y las enrumba hacia la obtención de lo que anhelan. Brophy (2004) por su parte, considera que la motivación es una construcción teórica para explicar el inicio, la dirección, la intensidad, la persistencia y la calidad de la conducta, especialmente el comportamiento dirigido a un objetivo. Desde esta perspectiva, los motivos son conceptos hipotéticos usados para explicar por qué la gente hace lo que hace, y se pueden considerar como necesidades o deseos que energizan a las personas para iniciar acciones propositivas.

En consonancia con este último planteamiento, Wolcott y Shattuck (2007) se refieren a las definiciones psicológicas que identifican dos tipos de motivación: intrínseca y extrínseca. La primera tiene un origen interno; un individuo obtiene satisfacción de realizar una determinada actividad, porque hace un llamado a sus valores, o responde a una necesidad o a un deseo personal. La motivación extrínseca está asociada con un beneficio recibido de una fuente diferente de la actividad misma. El individuo se mueve a la acción en respuesta a la posibilidad de un beneficio o recompensa externa. 
Hasta aquí y de acuerdo con lo propuesto, se ha caracterizado suficientemente la motivación, pudiéndose afirmar que todos los autores coinciden en que esta es el motor que impulsa a una persona a realizar determinadas acciones y a persistir en ellas hasta el cumplimiento de sus objetivos, término que se relaciona con voluntad e interés. Este motor está alimentado por motivos internos y externos que responden a necesidades y deseos personales, o a la búsqueda de recompensas externas. Pero, ¿qué papel juega esta energía en el proceso de aprendizaje en los estudiantes y, en particular, en los aprendices a distancia?

En el campo de la educación son importantes ambas formas de motivación. Según las teorías del aprendizaje (Tallon, 2005), para alcanzar un proceso satisfactorio e integral, es de vital importancia tanto la motivación interna como la externa, debido a que ambas se complementan y resultan relevantes en la obtención de resultados educativos óptimos. Estas mueven al estudiante a participar de forma activa en la dinámica de la clase, a realizar las actividades propuestas, a estudiar con las técnicas adecuadas, a investigar y a experimentar. Su motivación se basa en experiencias subjetivas de los mismos estudiantes, especialmente las vinculadas a su disposición de participar en las lecciones y actividades de aprendizaje y a sus razones para hacerlo (Brophy, 2004).

Urdan y Schoenfelder (2006) al respecto de motivación en el ambiente de clase, manifiestan que esta refleja una compleja interacción de numerosos factores personales y situacionales, muchos de los cuales están más allá del control de los educadores. Sin embargo, existen otros sobre los cuales los profesores pueden ejercer algún control: ayudar a los estudiantes a sentirse competentes, a que vean el aprendizaje como un proceso y no como un fin y a que satisfagan las necesidades que surjan de sus relaciones sociales. Algunos maestros tienen un don para impulsar a los estudiantes hasta altos niveles de motivación, de tal manera que los inspiran a trabajar más que al principio (Harmin y Toth, 2006). "Motivar para el aprendizaje, es mover al alumno a aprender, y crear las condiciones necesarias para lograrlo” (Guzmán García, 2007, 3).

Estas condiciones deben cumplirse tanto en el ambiente tradicional como en el ambiente a distancia. De acuerdo con Dennen y Bonk (2007), motivar a aprendices en línea es un desafío clave que enfrentan los instructores en la enseñanza superior. Este hecho se agudiza, teniendo en cuenta las observaciones planteadas por ellos mismos sobre las quejas frecuentes de los instructores de ambientes de aprendizaje en línea, respecto a las tasas de deserción y los bajos niveles de participación de los estudiantes en actividades de curso.

Aquí es claro que la motivación de aprendices de cursos en línea es clave para resolver los problemas antes mencionados y que para lograrlo es necesario el concurso definitivo del profesor y la disponibilidad de herramientas pertinentes en línea. Sin embargo, como muy bien lo expresan Dennen y Bonk (2007), los instructores no solo deben conocer y manejar los tipos de herramientas 
colaborativas en línea para promover la participación de los estudiantes, sino también saber cómo integrar una pedagogía eficaz cuando las tecnologías sean débiles. Entonces, también cobra importancia el papel del profesor para establecer la relación adecuada entre la motivación y el aprendizaje en la construcción del conocimiento, adaptando diferentes actividades de aprendizaje y herramientas tecnológicas para lograr dicha participación.

De acuerdo con Dennen y Bonk (2007), revisando la investigación sobre motivación, se han encontrado ciertas estrategias claves, consistentemente efectivas en ambientes de clases convencionales. Por ejemplo, los instructores eficientes crean un ambiente de apoyo desafiante que proyecta entusiasmo, ofrece elección, crea metas de corto plazo y ofrece realimentación inmediata sobre rendimiento. Además pueden intentar estimular la curiosidad, el control y la fantasía del estudiante. Según los autores, deberían diseñar un contenido personal y concreto, mediante el uso de tareas de aprendizaje relevantes y auténticas, y permitir a los estudiantes crear y mostrar los productos finales. Naturalmente, los instructores deben fomentar la interacción entre compañeros, impulsar actividades divertidas y a manera de juegos, integrar estructura y flexibilidad en las asignaciones e incluir actividades que presenten divergencias o conflictos.

Estas actividades de aprendizaje incorporan estrategias pedagógicas, diseñadas con el objeto de mejorar la calidad de la enseñanza en línea, ayudar al rendimiento de los estudiantes y promover debates, discusiones y reflexiones en línea (Bonk \& Dennen, 2007). Tales actividades se relacionan con la creatividad, el pensamiento crítico, el aprendizaje cooperativo y la motivación. Las actividades instruccionales que involucran estrategias pedagógicas orientadas a motivar a los estudiantes para participar y mejorar su rendimiento, son conocidas como actividades motivacionales. Estas estrategias pueden y deben ser utilizadas en cualquier momento durante el proceso de enseñanza-aprendizaje, con el fin de inyectar o alimentar en el estudiante, el ánimo y el entusiasmo que por razones intrínsecas o extrínsecas, puedan encontrarse en un mínimo nivel, y para mejorar el clima de desarrollo de la instrucción.

Se han propuesto diferentes actividades pedagógicas de aprendizaje en línea, de las cuales Bonk y Dennen (2007) presentan una amplia relación. Sin embargo, con el fin de ilustrar las actividades orientadas hacia la motivación, para la elaboración de este documento se tuvo en cuenta el trabajo presentado por Dennen y Bonk (2007), el cual hace una amplia referencia a actividades en línea y a los diferentes elementos motivacionales que ellas orientan.

Dentro de las actividades presentadas por estos autores, vale la pena mencionar: rompehielos, autoevaluación, encuestas y sondeos, casos, lluvia de ideas, charlas electrónicas, debates y juego de roles, realimentación y comentarios críticos de amigos y compañeros, y visitas a galerías. Los elementos motivacionales orientados en mayor o menor grado por estas actividades se refieren a: clima y 
tono, realimentación, participación, pertinencia, elección, variedad, curiosidad, tensión, interacción entre compañeros y objetivo impulsado. La relación de cada actividad con el elemento motivador, así como sus características y ejemplos de aplicación, pueden consultarse en el artículo "We'll leave a light on for you: keeping learners motivated in online courses" (Dennen \& Bonk, 2007).

La implementación de estas actividades dentro de ambientes de aprendizaje en línea, es esencial para el éxito de este proceso. La mayoría de ellas pueden realizarse en forma sincrónica y asincrónica y adaptarse a cualquier disciplina del conocimiento. Además, de acuerdo con Dennen y Bonk (2007), si bien es cierto que algunas parecen adaptarse mejor a la interacción en vivo, otras pueden ser más fructíferas cuando los estudiantes se benefician de un período de tiempo prolongado para poder participar y reflexionar, como lo permiten las tecnologías asincrónicas.

\section{Conclusiones}

Esta reflexión, realizada en torno a la motivación, las actividades motivacionales y su papel en el ámbito educativo, induce a pensar en la importancia de esta fuerza que mueve a realizar actividades y, por consiguiente, a alcanzar las metas propuestas. Los avances, descubrimientos y grandes logros de la humanidad en todas las disciplinas del saber, se han conseguido gracias a la decisión de hacer algo, a la perseverancia para mantenerse en el proyecto el tiempo que sea necesario y a la voluntad para finalizarlo y hacerlo de público conocimiento en todo el mundo. Indiscutiblemente, para trazarse objetivos y lograr su realización, los seres humanos deben estar motivados.

En el proceso educativo es innegable la importancia que reviste el concepto de motivación para el logro de un aprendizaje significativo. De acuerdo con Díaz y Hernández (1998), este logro está relacionado con la necesidad de inducir en el estudiante el interés y el esfuerzo necesarios para su consecución, por lo que cualquier modelo educativo debe incorporar diversos principios en torno a la motivación. Los teóricos del aprendizaje y los profesores están de acuerdo en que los estudiantes motivados aprenden con mayor rapidez y más eficazmente que los que no lo están. Pero este estado debe permanecer durante todo el proceso educativo, desde su inicio, durante su transcurso y hasta su finalización.

En la educación a distancia el interés se orienta hacia la concreción de un aprendizaje autónomo, autodirigido y autorregulado; igualmente debe dar respuesta a problemas de deserción y baja participación de los aprendices, evitándoles desistir de alcanzar sus metas por falta de esfuerzo y perseverancia. Los mayores niveles de compromiso y rendimiento, son alcanzados por las personas con fuertes objetivos de aprendizaje (Molden \& Dweck, 2000). El papel 
del profesor en la metodología a distancia es el de un facilitador del proceso de aprendizaje y el de un motivador para promover el compromiso, la participación y el rendimiento de los estudiantes.

Para lograrlo de una manera eficiente, el profesor necesita utilizar recursos y estrategias atractivas adaptadas a las necesidades de los aprendices. En especial, las actividades en línea que orientan elementos motivadores, son esenciales en el éxito de los ambientes de aprendizaje a distancia. Tanto para ambientes en línea, como para ambientes cara a cara, los instructores deben buscar espacios claves donde se puedan orientar cómodamente estas actividades, dentro del diseño curricular. Lo importante aquí es enfocarse hacia el elemento motivacional y sus principios, y no solamente en el rango de las posibles tareas, ya que las actividades son simples vehículos a través de los cuales la motivación y el aprendizaje efectivo tienen lugar (Dennen \& Bonk, 2007).

Para finalizar, se presenta un aparte del capítulo 1 de Brophy, (2004): "Student Motivation: The Teacher's Perspective", que permite reflexionar sobre el quehacer del profesor y el método de instrucción:

Aprender es divertido y emocionante, al menos cuando el plan de estudios está bien adaptado a los intereses y habilidades de los estudiantes y el profesor hace hincapié en actividades prácticas. Al enseñar las cosas de la manera correcta, la motivación toma lugar por sí misma. Si los estudiantes no están disfrutando del aprendizaje, algo está mal con el currículo y la instrucción; pasó de alguna manera de ser una actividad inherentemente agradable, a ser un trabajo tedioso.

\section{Referencias bibliográficas}

Boyle, F., kwon, J., Ross, C. \& Simpson, O. (2010). Student-student mentoring for retention and engagement in distance education. Open Learning: The Journal of Open and Distance Learning, 25(2), 115-130.

Bonk, C. J. \& Dennen, P. V. (2007). Frameworks for design and instruction. En M. G. Moore (Ed.), Handbook of distance education (pp. 233-246). Mahwah, NJ: Lawrence Erlbaum Associates, Publishers.

Brophy, J. E. (2004). Motivating students to learn. Mahwah, NJ: Lawrence Erlbaum Associates, Publishers. Recuperado el 14 de abril de 2010, de http://www.netlibrary. com. ezproxylocal.library.nova.edu/Reader/

Dennen, V. P. \& Bonk, C. J. (2007). We'll leave a light on for you: keeping learners motivated in online courses. En B. H. khan (Ed.), Flexible learning in an information 
society (pp. 64-74). Hershey, PA: The Idea Group Inc. Recuperado el 30 de marzo de 2010, de http://www.netlibrary.com.ezproxylocal.library.nova.edu/Reader/Díaz, F. \& Hernández, G. (1998). Estrategias docentes para un aprendizaje significativo. México, D. F.: Mc Graw Hill Interamericana Editores, S. A.

Facundo, A. H. (2009). Análisis sobre la deserción en la educación superior a distancia y virtual: El caso de la UNAD - Colombia. Revista de Investigaciones UNAD, 8(2), 117-149.

Gunawardena, Ch. N. \& Mclsaac, M. S. (2004). Distance Education. En D. H. Jonassen (Ed.), Handbook of research on educational communications and technology. Mahwah, $\mathrm{NJ}$ : Erlbaum. Recuperado el 7 de enero de 2010, de http://www.netlibrary.com. ezproxylocal. library.nova.edu/

Guzmán García, G. J. (2007). Definición, características y elementos de la motivación. Recuperado el 20 de marzo de 2010, de http://www.monografias.com/trabajos61/ motivacion-caracteristicas/motivacion-caracteristicas.shtml

Harmin, M. \& Toth, M. (2006). Inspiring active learning: A complete handbook for today's teachers. Alexandria, Va: Assoc. for Supervision and Curriculum Development. Recuperado el 14 de abril de 2010, de http://www.netlibrary.com.ezproxylocal.library. nova.edu/Reader/

Isaza, J. F. (2009). Deserción en la Educación superior. El Observatorio de la Universidad Colombiana. Recuperado el 29 de junio de 2010, de http://www.universidad.edu.co/ index.php?option=com_content\&view=article\&id=689:desercion-en-la-educacionsuper ior\&catid=12:opini\&/temid=200

Ministerio de Educación Nacional. (2009). Sistema de Prevención y Análisis de la Deserción en las Instituciones de Educación Superior en Colombia. Recuperado el 9 de junio de 2010, de http://www.mineducacion.gov.co/1621/article-156292.html

Molden, D. C. \& Dweck, C. S. (2000). Meaning and motivation. En C. Sansone \& J. M. Harackiewicz (Eds.), Intrinsic and extrinsic motivation: The search for optimal motivation and performance (pp. 131-141). San Diego: Elsevier.

Nichols, M. (2010). Student perceptions of support services and the influence of targeted interventions on retention in distance education. Distance Education, 31(1), 93-113.

Simonson, M., Smaldino, S., Albright, M. \& Zvacek, S. (2009). Teaching and learning at a distance: Foundations of distance education. Boston: Pearson Education Inc.

Simpson, O. (2004). The impact on retention of interventions to support distance learning students. Open Learning: The Journal of Open and Distance Learning, 19(1), 79-95. 


\section{Ana Ilva Capera Urrego}

Actividades motivacionales para aprendices en línea, artículo producto de la investigación

Tallon, P. (2005). La motivación como estrategia de aprendizaje. Recuperado el 14 de abril de 2010, de http://www.diariocordoba.com/noticias/noticia.asp?pkid=172881

Urdan, T. \& Shoenfelder, E. (2006). Classroom effects on student motivation: Goal structures, social relationships, and competence beliefs. Journal of School Psychology, 44, 331-349.

Wolcott, L. L. \& Shattuck, k. (2007). Faculty participation: Motivations, incentives, and rewards. En M. G. Moore (Ed.), Handbook of distance education (pp 377-390). Mahwah, NJ: Lawrence Erlbaum Associates, Publishers.

Woolfolk, A. E. (1999). Psicología educativa. México: Prentice Hall Hispanoamericana. Yukselturk, E., \& Inan, F. A. (2006). Examining the factors affecting student dropout in an online learning environment. Presentación en línea. (No. de servicio de reproducción de documentos ERIC ED 494 345) 